\title{
Intergenerational Transmission of Stress in Humans
}

\author{
Mallory E Bowers ${ }^{1}$ and Rachel Yehuda*,1,2,3 \\ ${ }^{1}$ Department of Psychiatry, Icahn School of Medicine at Mount Sinai, NY, NY, USA; ${ }^{2}$ Mental Health Care Center, James J. \\ Peters Veterans Affairs Medical Center, Bronx, NY, USA; ${ }^{3}$ Department of Neuroscience, Icahn School of Medicine at Mount, \\ NY, NY, USA
}

The hypothesis that offspring are affected by parental trauma or stress exposure, first noted anecdotally, is now supported empirically by data from Holocaust survivor offspring cohorts and other populations. These findings have been extended to less extreme forms of stress, where differential physical, behavioral, and cognitive outcomes are observed in affected offspring. Parental stress-mediated effects in offspring could be explained by genetics or social learning theory. Alternatively, biological variations stemming from stress exposure in parents could more directly have an impact on offspring, a concept we refer to here as 'intergenerational transmission', via changes to gametes and the gestational uterine environment. We further extend this definition to include the transmission of stress to offspring via early postnatal care, as animal studies demonstrate the importance of early maternal care of pups in affecting offsprings' long-term behavioral changes. Here, we review clinical observations in offspring, noting that offspring of stress- or trauma-exposed parents may be at greater risk for physical, behavioral, and cognitive problems, as well as psychopathology. Furthermore, we review findings concerning offspring biological correlates of parental stress, in particular, offspring neuroendocrine, epigenetic, and neuroanatomical changes, in an attempt to determine the extent of parental stress effects. Although understanding the etiology of effects in offspring is currently impeded by methodological constraints, and limitations in our knowledge, we summarize current information and conclude by presenting hypotheses that have been prompted by recent studies in the field.

Neuropsychopharmacology Reviews (2016) 41, 232-244; doi:I 0.I038/npp.2015.247; published online 2 September 2015

\section{INTRODUCTION}

Severe stress exposure in a parent-the kind that can result in mental disorders such as depression, anxiety, or post-traumatic stress disorder (PTSD) - is a risk factor for a number of adverse outcomes, including psychopathology, in offspring. The mechanism(s) through which this risk is conferred, however, have not been fully elucidated. For instance, stress-exposed parents may confer vulnerability via genetic risk factors (ie, their offspring may inherit the same or similar genetic risks that have an impact on their own stress vulnerability), or through behavioral alterations stemming from the development of stress-related psychopathology (ie, affecting their ability to parent or the childhood environment of the offspring). In recent years, as a result of advances in the understanding of epigenetic mechanisms, an additional hypothesis has been promulgated-that offspring of severely stress-exposed

*Correspondence: Professor R Yehuda, Department of Psychiatry, Icahn School of Medicine at Mount Sinai, James J. Peters Veterans Affairs Medical Center, 526 OOMH PTSD 116/A, JJP VAMC, 130 W Kingsbridge Road, Bronx, NY 10468, USA, Tel: +718 741 4000, ext. 6964, Fax: +718 741 4703, E-mail: rachel.yehuda@va.gov

Received 1 May 2015; revised 19 July 2015; accepted 20 July 2015; accepted article preview online 17 August 2015 parents are at risk for adverse outcomes because of enduring epigenetic changes in parental biological systems that have arisen in response to stress exposure and are transmitted (Yehuda and Bierer, 2009). This process has been referred to as 'intergenerational transmission'.

Here, we present evidence for the phenomenon of intergenerational transmission, particularly focusing on biological correlates in the second generation, to better understand the mechanism(s) of transmission from parent to offspring. We begin this review by discussing the field's progression in understanding regarding stress and its long-lasting marks, ie, via PTSD and intergenerational effects. We note affected offspring clinical features, from physical, behavioral, cognitive, and psychological, as well as biological correlates, including neuroendocrine, epigenetic, and neuroanatomical changes.

Throughout this discussion, we attempt to highlight studies investigating intergenerational transmission in the aftermath of stress exposure rather than transmission of parental psychopathology to offspring. By emphasizing this distinction, it is our intention to clarify that effects of parental stress and effects of parental psychopathology may be differentially transmitted to offspring. Note, however, that numerous reports refer to parental stress, anxiety, and depression as if these were interchangeable constructs. 
Furthermore, we attempt to distinguish between stress exposures that occurred before conception, at the time of conception, at the time of pregnancy, or in the early postnatal period, where possible. A limitation of the current literature is that it has particularly focused on the effects of maternal stress/trauma exposure on offspring, especially during pregnancy, and thus the current review preferentially explores intergenerational transmission of stress from this perspective. Moreover, while we incorporate discussion of seminal animal studies that clarify the mechanistic understanding of intergenerational transmission of stress, we restrict this review to the examination of human studies.

Understanding intergenerational transmission of stress, particularly its underlying mechanism(s), is complex, given the challenges related to a consensus on operational definitions and methodological problems. We attempt to clarify the inherent problems of studying intergenerational effects in humans by proposing a framework that distinguishes between offspring effects resulting from parental stress $v s$ those resulting from parental psychopathology, as well as proposing additional methodological approaches and future directed lines of inquiry.

\section{CONCEPTUAL FRAMEWORK}

Stress theory posits that organisms will mount, and continue to express, a biobehavioral response to an environmental challenge as long as the challenge or stressor is present (Selye, 1956). Removal of the stressor triggers biological responses that restore homeostasis, promoting a return to baseline functioning. Activation of the stress response for short periods of time does not result in long-term recalibration of the stress response axis, whereas chronic stress can exert effects that may be quite long lasting. On the basis of a model of the acute stress response, removal of the stressor will result in the engagement of numerous biological and psychological mechanisms in the interest of restoring neural, physiological, and behavioral systems that were activated in response to the challenge (Yehuda, 2002).

The idea that stress effects may be prolonged and remain present even after the active threat is no longer present is one that appears to challenge the paradigm of homeostasis upon which stress theory is based. Moreover, the concept of intergenerational transmission of stress effects relies on the observation, based on animal and human studies, that stress may induce long-lived and widespread effects in the parent. It is fair to credit the establishment of the diagnosis of PTSD in 1980 as a key driver of inquiry into intergenerational transmission of stress. Before the advent of this diagnosis, there was a gap in the psychiatric and stress literature with respect to the conceptualization of chronic effects of trauma. The diagnosis of PTSD filled this gap in nosology and theory by asserting that behavioral and biological consequences associated with exposure to extreme trauma could continue to exert their effects long after exposure to the event. Prior formulations in psychiatry would have conceptualized mental health responses that do not abate as manifestations of constitutional (ie, genetic or temperamental) factors (Yehuda and McFarlane, 1995).

Awareness of PTSD led the scientific and lay public to understand that trauma exposure leaves enduring marks, even if those include positive and transformative effects, as well as mental health symptoms, the emergence of maladaptive cognitions, personality, and other behavioral changes. For the last two decades, scientists have been concerned with addressing the question of what sustains long-term effects. Advances in molecular biology and neuroscience have enhanced our understanding of the mechanisms by which the effects of traumatic stressors may persist.

It can also be asked whether and how effects of trauma can be passed to the next generation. Impetus for this line of research has come from offspring of trauma survivors who have themselves used a variety of forums, including the arts and the literature, as well as mental health clinics, to articulate the effects of parental trauma on their own mental health outcomes. Offspring of trauma survivors have described vicarious traumatization through the stories told by exposed parents, their own feelings of helplessness, and burdens of compensating not only for parental losses but parental damage (Spiegelman, 1991). Science, in parallel, has made strides in understanding offspring effects as they are manifested in people and laboratory animals. As a result, it now possible to at least conceptually distinguish between effects that are present in the parent as a function of stress and then appear to also exist in some form in unaffected offspring, and effects that may be present in the offspring as a result of parental stress exposure even if the parent does not demonstrate the consequence per se (ie, the adaptive response of the offspring to trauma effects in the parent).

\section{WHAT IS THE INTERGENERATIONAL TRANSMISSION OF STRESS?}

Intergenerational transmission refers to an effect in offspring as a result of parental exposures and characteristics that is more specific than the generally observed link between parental problems and offspring outcomes. Parents can model behaviors, and children can 'learn' to react to their environments in a manner similar to their parents without necessarily invoking molecular explanations of intergenerational transmission. Even learning theory could explain why children exhibit behavior similar to their parents (Bandura, 1977). Furthermore, phenotypic changes in offspring can occur as a consequence of parental deficits, such as in child rearing (Vostanis et al, 2006). Moreover, many offspring report experiencing parental trauma vicariously or imagining the traumatic events that they know were experienced by their parents. All of these can affect offspring without the effect being transmitted directly from parent to child. The observation in offspring of biological changes associated with trauma in the parent (potentially in the 
Fo

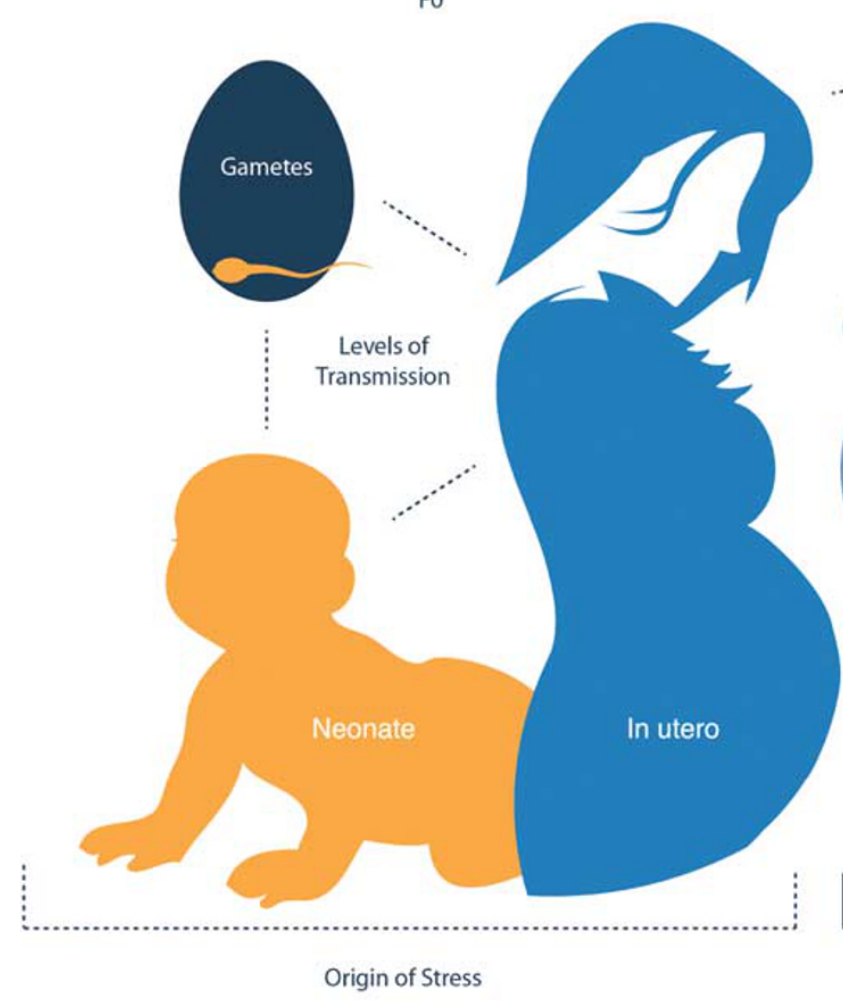

F1

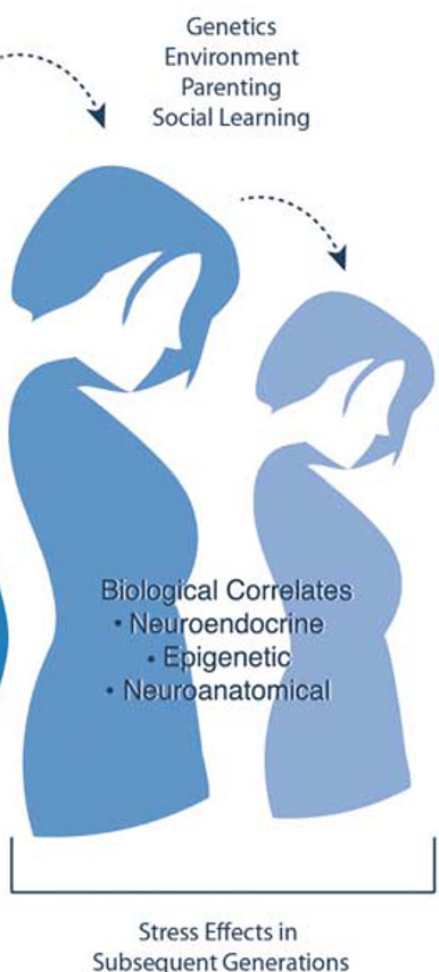

Figure 1. Parental stress can be transmitted via gametes, the gestational uterine environment, and early postnatal care. Across species, studies suggest that the effects of parental stress can be directly transmitted to offspring via gametes, uterine environment during pregnancy, or during early postnatal care of newborns. In studies of Holocaust survivor offspring, parental trauma occurred years before conception, suggesting that effects in offspring might be due in part to some biological change in gametes. This is supported by rodent experiments, which demonstrate epigenetic and microRNA changes in sperm of stressed fathers. A number of studies also observe effects in offspring associated with maternal stress during pregnancy. Moreover, animal studies demonstrate that variations in early maternal care results in long-lasting neural, hormonal, cognitive, and behavioral changes in pups. Genetics, parenting, social learning, and shared environmental context can also influence outcomes related to stress across generations. Stress effects that are inherited via an 'intergenerational transmission' mode are reflected in offspring biological changes, including neuroendocrine, epigenetic, and neuroanatomical changes.

absence of offspring trauma) may be an indication of similar genetic risks in both generations, rather than an indication of intergenerational transmission of the biological effect. This type of 'transmission' accords more with a traditional model of genetics, but is not necessarily explanative of transmittable stress effects.

The more novel and intriguing idea that an observed change in offspring may be a consequence of a biological change in the parent that occurred in response to a traumatic event was initially based on results from large cohorts examining the effects in offspring of pregnant women exposed to the stress of starvation during the Dutch famines (Barker, 1990, 1998). These studies yielded several examples of what can be described as 'intergenerational transmission', where offspring were directly affected by maternal nutritional deprivation, separate from stress-related alterations in maternal behaviors.

Observations from the Dutch Famine study and others have demonstrated that parental stress may be transmitted via the gametes (Franklin et al, 2010; Dias and Ressler, 2014; Gapp et al, 2014) or the gestational uterine environment (Yehuda et al, 2005). More recently, animal work has demonstrated that variations in early maternal care (originally induced by the stress of removing mothers from pups and briefly handling the mothers before returning them to the home cage) results in altered neural, hormonal, cognitive, and behavioral responses in pups, that were not directly manipulated except for the brief separation from the mother, and the mother's behavior upon return (Liu et al, 1997; Caldji et al, 1998; Weaver et al, 2004). Although maternally mediated effects in offspring have been the subject of primary investigation, it should be mentioned that paternal stress also affects offspring, presumably through effects on sperm or early paternal care (Franklin et al, 2010; Dias and Ressler, 2014; Gapp et al, 2014). Here, we refer to parent biological changes resulting from stress or psychopathology that have an impact on their gametes, gestational uterine environment or early postnatal care to alter offspring biology, and thus offspring outcomes, as 'intergenerational transmission'. Figure 1 depicts the three levels at which biological effects of stress in parents are thought to directly have an impact on offspring. We also note other mechanisms, including genetics, social learning, parenting, and shared environmental contexts, that can 
induce stress across generations. Stress effects that are inherited via an 'intergenerational transmission' mode are reflected in offspring biological changes, including neuroendocrine, epigenetic, and neuroanatomical changes.

\section{CLINICAL OBSERVATIONS AND SIGNIFICANCE}

Studies consistently demonstrate that offspring of extremely stressed or traumatized parents are at higher risk for mental and physical adverse outcomes. This has been demonstrated in instances where exposure predated conception or exposure occurred during pregnancy, supporting the idea that transmission can occur via gametes and/or the fetus. For example, adult offspring of Holocaust survivors were shown to be at greater risk for the development of PTSD, depression, and anxiety disorders (Yehuda et al, 2008). This was true, even though in all cases offspring were born after World War II. It has also been shown that women who develop PTSD as a result of trauma exposure during pregnancy-such as having to evacuate the World Trade Center on 9/11-give birth to affected offspring with evidence of a trimester effect (Yehuda et al, 2005). The greater influence of maternal exposure during the third compared with second trimester provides evidence for the relevance of in utero effects to the transmission of biological risk.

Regarding offspring physical effects, maternal PTSD and maternal prenatal stress, including psychosocial stress, maltreatment, and exposure to a terrorist attack, have been found to associate with impaired uterine blood flow, low birth weight, and pre-term birth (Wadhwa et al, 1993; Glover, 1997; Berkowitz et al, 2003; Lederman et al, 2004, Coussons-Read et al, 2012; Cederbaum et al, 2013; Yonkers et al, 2014; Christiaens et al, 2015). Impaired uterine blood flow, low birth weight, and pre-term birth, in turn, have been linked with the subsequent development of hypertension, insulin resistance, Type 2 diabetes, and cardiovascular disease in adult offspring (Barker, 1998). Similarly, parental Holocaust exposure has also been associated with hypertension, dyslipidemia, Type 2 diabetes, and subjective health ratings in offspring (Flory et al, 2011).

Aside from physical health problems, behavioral and emotional issues have also been observed in affected offspring. Holocaust survivor offspring were found to exhibit higher anxiety, lower self-esteem, and inhibition of aggression (Gangi et al, 2009; Flory et al, 2011). Similar effects have been observed in offspring of prenatally stressed mothers, in whom anxiety in the second and third trimesters was associated with offspring depressive symptoms and behavioral/ emotional problems, including conduct problems, emotional problems, and hyperactivity/inattention ( $\mathrm{O}^{\prime}$ Connor et al, 2002; Van den Bergh et al, 2008).

Offspring of mothers who experienced stress while pregnancy may also experience difficulties in cognitive domains. It is hypothesized that high levels of stress can affect offspring brain development, which may be reflected by changes in cognitive abilities. Adolescent offspring of mothers who experienced high levels of anxiety during weeks 12-22 of their pregnancy responded more impulsively and had difficulties organizing their cognitive resources (Van den Bergh et al, 2005; Mennes et al, 2006) High levels of maternal anxiety during gestation have been associated with lower inhibitory control in girls on a Flanker task and lower visuospatial working memory performance in boys and girls on a sequential memory task (Buss et al, 2011). Toddlers of mothers who experienced higher levels of objective stress exposure while pregnancy during the 1998 Québec ice storm exhibited lower intellectual abilities (as measured by Bayley Mental Development Index) and productive language abilities than toddlers of mothers who experienced lower levels of objectively defined prenatal stress.

Behavioral/emotional problems and possibly cognitive deficits may explain vulnerability of offspring to psychopathology. In a foundational study, Solomon et al (1988) observed that Holocaust survivor offspring had higher levels of PTSD symptoms after serving in the military during wartime, despite being physically and psychologically healthy before combat experience. Similarly, separate studies have also observed increased vulnerability to PTSD and other psychiatric disorders, including depression, among offspring of Holocaust survivors (Yehuda et al, 1998, 2001). Lifetime maternal PTSD symptoms have also been shown to dosedependently associate with offspring PTSD symptoms in the general population (Roberts et al, 2012). Comparable findings have been observed in studies of offspring prenatally exposed to nuclear and natural disaster, where offspring of affected parents exhibited greater rates of depression and attention deficit hyperactivity disorder symptoms (Watson et al, 1999; Huizink et al, 2007).

Altogether, offspring outcomes, including physical, behavioral, cognitive, and psychiatric effects that were initially noted anecdotally have now been documented empirically across a number of studies. Observed clinical effects have prompted further study into their biological origins. A variety of these biological changes in offspring have now been found to associate with symptoms and exposures in parents, supporting an intergenerational mode of transmission.

\section{METHODOLOGICAL PROBLEMS}

A major difficulty related to the study of intergenerational transmission is determining whether offspring outcomes are mediated by the severity of stressor exposure to the parent, or the resultant physical or biobehavioral consequences of the stressor. For this reason severity of parental exposures and parental symptoms should be carefully studied, and not assumed to reflect the same parameters. Studies investigating the etiology of stress transmission will benefit from a clarification in language and terminology. The term 'intergenerational transmission' has been used liberally in 
the literature to refer to genetics, social learning, or the other processes by which offspring are directly affected by changes to parent biology. To date, it has been difficult to distinguish between parental biology and parental behavior as mediators of offspring effects. These classifications and the accompanying use of appropriate measures defining stressors or stress responses, including psychopathology, are pivotal for the rigorous interpretation of data. Some studies have attempted to address this discrepancy in language. For instance, in a study examining the impact of prenatal maternal stress associated with the 1998 Québec ice storm on offspring development, Laplante et al (2004) attempted to assess 'objective' stress via mothers' responses to questions regarding charecteristics of exposure to the storm, including threat, loss, scope, and change. Subjective stress was assessed using the Impact of Event Scale-Revised, which asked participants to rate symptoms from three categories relevant to post-traumatic stress disorder (PTSD): intrusive thoughts, hyperarousal, and avoidance. To the extent that these domains are separate, using measures that distinguish objective and subjective stress, and furthermore stress and psychopathology, will better enable researchers to identify effects in offspring that are mediated by parent biology in response to stress.

Methodologically, a key obstacle common to studies of intergenerational stress is the fact that parents and offspring often share a living environment and, thus, share similar constant, long-term environmental stressors, eg, poverty. Disentangling parent/offspring shared environmental stressors from transmitted stress is likely to be complex. Environmental stress may simultaneously have an impact on parent and offspring functioning, just parenting functioning (which is then subsequently transmitted to offspring), or directly affect offspring functioning. This issue further underlines the need to delineate stress from psychopathology, as certain psychiatric disorders increase the likelihood of experiencing additional stressors (Hammen, 1991). Interpreting the effect of transmitted environmental stress on offspring is further complicated by that fact that a number of 'environmental' variables are, at least in part, genetically mediated (O'Connor et al, 1995; Jerskey et al, 2010).

In addition, the problem with noting manifestations of parental stress in offspring is the difficulty in determining at what stage parental stress effects were transmitted-whether observed effects are due to changes to the gamete of the stress-exposed parent, changes within the uterine environment, or effects resulting from variations in neonatal care. Sometimes traumatic exposures that occur before conception can exert effects in later periods as a result of a continuation of PTSD or other mental health symptoms. In addition, it is often the case that persons experience extreme stress more than once or in a continuous manner. Although it is evident at what stage stress is transmitted when the stressor occurs as a discrete event, eg, natural disaster, detailed participant histories would identify additional forms of parental stress that could contribute to offspring outcomes.
A major limitation of the current literature is that it has preferentially focused on the effects of maternal stress/trauma exposure on offspring, particularly during pregnancy, to the exclusion of paternal effects. Paternal stress may directly affect offspring via epigenetic modifications in sperm (similar to changes in DNA methylation or microRNA content observed in rodent models; Franklin et al, 2010; Dias and Ressler, 2014; Rodgers et al, 2013; Gapp et al, 2014) or early postnatal care; however, paternal stress may indirectly have an impact on offspring via effects on the mother/child-rearing partner, additionally. Likely, paternal stress is transmitted by a combination of both direct (ie, sperm) and indirect (ie, mother/child-rearing partner) modes. Studies investigating offspring effects of paternal stress may be instrumental in determining whether paternal effects are unique from maternal effects, as well as whether combined effects of maternal and paternal stress are additive. In studies of Holocaust survivor offspring, maternal, not paternal, PTSD has an effect on offspring PTSD; however, paternal PTSD moderates the effect of maternal PTSD in that the effect of the mother's PTSD is greater in the presence of paternal PTSD (Yehuda et al, 2008). Studies that account for stress in both mothers and fathers will enable researchers to more thoroughly interpret effects in offspring.

Consequently, determining the etiology of stress transmission, in particular, the interactions between, directionality of, and independence of factors contributing to observed effects in offspring is difficult. This is compounded by a lack of clarity regarding jargon specific to studies investigating intergenerational transmission of stress. In this regard, consensus on operational definitions common to the field could enable better identification of factors originating in parents that are responsible for mediating offspring outcomes. Methodologically, the use of conservative statistical tests, large cohorts of subjects, and identified gold standard methods of data collection will reduce the incidence of false positives. As mentioned, detailed participant histories, in both parents and offspring, will illuminate all potential contributions of stress to offspring outcomes, besides the most obvious instances of extreme stress. Animal studies will also continue to be instrumental in delineating factors contributing to the etiology of biological and behavioral observations in affected offspring, particularly in determining differences resulting from whether stress was transmitted at the level of the gamete, the uterine environment, or during the period of neonatal care.

\section{BIOLOGICAL CORRELATES IN OFFSPRING}

Investigation into the biological correlates of intergenerationally transmitted stress is a relatively recent area of inquiry, with most research dedicated to the investigation of hypothalamic-pituitary-adrenal (HPA)-axis dysfunction in the offspring of parents influenced by stress. This area of research has now extended to the investigation of epigenetic modifications as well as neuroanatomical changes in affected 
TABLE 1 Neuroendocrine Correlates of Parental Stress and Parental Cortisol in Offspring

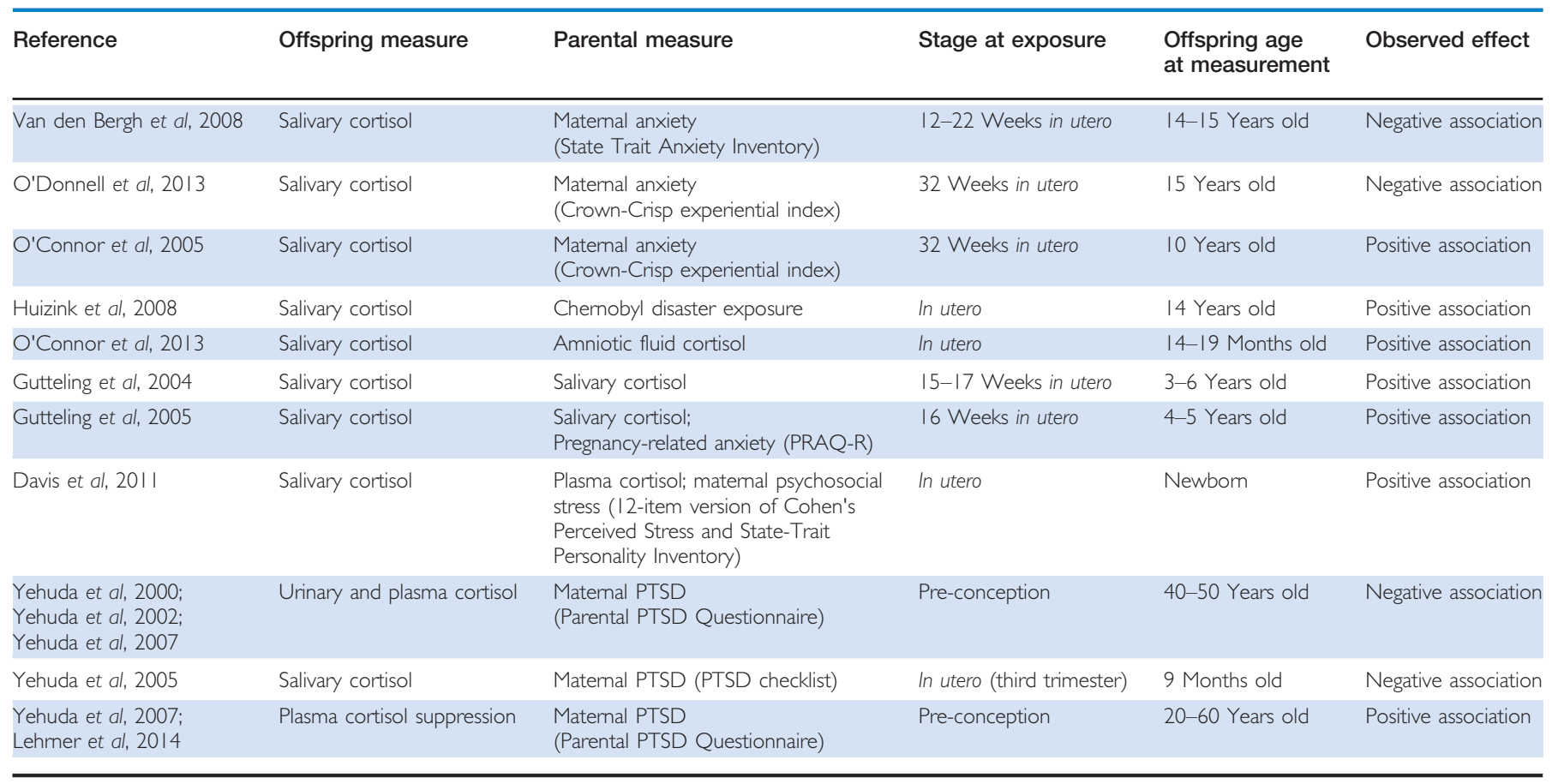

offspring. Although a number of biological correlates have been uncovered, the mechanism(s) involved in transfer of stress from one generation to the next are still unknown, and are likely to be numerous and complex. Cortisol is one appealing candidate, as evidence suggests that circulating cortisol accesses gametes and the gestating fetus (Graves and Eiler, 1979; Stratholt et al, 1997; Gitau et al, 1998). Furthermore, cortisol, as a part of the glucocorticoid receptor (GR) complex, affects gene transcription and could, ultimately, permanently alter the offspring epigenome (Stahn et al, 2007). Intriguingly, recent evidence also suggests that microRNA, which could itself be regulated by cortisol and has been shown to be regulated by stress, may have a role in intergenerational inheritance (Cortez et al, 2011; Daxinger and Whitelaw, 2012; Honda et al, 2013; Issler and Chen, 2015).

\section{Neuroendocrine}

A number of studies have measured maternal prenatal stress and subsequent offspring basal HPA-axis activity, primarily assessed via concentration of the stress hormone cortisol in blood. For a summary of the reviewed studies observing neuroendocrine changes in affected offspring, refer to Table 1. Stress-induced activation of the HPA-axis results in increased production and release of cortisol by the adrenal gland. Increased cortisol mobilizes resources needed to engage the flight or fight response, and is part of a complex and coordinated series of biological responses to stress. Stress-related increases in cortisol eventually result in an inhibition of HPA axis and other stress-activated systems, terminating the stress response (Yehuda, 2002). Sustained exposure to a stressor results in dysregulation of the HPA-axis reflected by changes in circulating levels of cortisol at baseline and in response to stress (McEwen, 2006).

In PTSD, there appears to be a premature termination of the cortisol response at the time of a traumatic event because of enhanced GR sensitivity that results in a failure to contain the sympathetic nervous system response, leading to catecholamine dysregulations (Yehuda, 2002). Lower than normal cortisol levels persist in PTSD, and it may be that cortisol levels were lower in persons who developed PTSD even before they were exposed to trauma (Yehuda et al, 2000; Delahanty et al, 2000; Pineles et al, 2013). That is, there is some evidence that low cortisol levels are risk factors for PTSD. This hypothesis was supported by data demonstrating that parental, and specifically maternal, PTSD associated with lower cortisol levels in offspring (Yehuda et al, 2000). Importantly, these same offspring were found to be at higher risk for PTSD (Yehuda et al, 2008). The idea that low cortisol levels may be related to PTSD vulnerability, potentially due to the presence of parental PTSD acting as a risk factor, was bolstered by additional studies that replicated low cortisol findings in offspring, even when accounting for offspring's own traumatization and PTSD (Yehuda et al, 2002, 2007). The offspring in these studies were conceived after direct parental exposure to the Holocaust, highlighting the idea that accompanying changes to parental biology associated with trauma exposure and PTSD can change offspring biology, thus predisposing offspring to specific outcomes. Follow up studies found that Holocaust exposure itself was associated with offspring neuroendocrine changes, demonstrating that 
stress effects in response to trauma, and not just psychopathology, can be transmitted (Bierer et al, 2014). These studies have proven foundational for the investigation of neuroendocrine correlates in offspring related to parental stress.

Several studies investigating maternal prenatal stress have also found corresponding changes in offspring cortisol levels, although the relationship between parental stress, parental cortisol, offspring stress, and offspring cortisol is less clear compared with the PTSD literature. Maternal anxiety at 12-22 weeks gestation was associated with lower awakening cortisol and higher levels in the evening 14-15-year-old offspring (Van den Bergh et al, 2008). Similarly, maternal anxiety at 32-week gestation was associated with reduced cortisol-awakening response and flatter diurnal slope in offspring of 15 years of age (O'Donnell et al, 2013). Conversely, maternal anxiety at 32-week gestation was positively associated with awakening cortisol levels in offspring at 10 years old (O'Connor et al, 2005). This positive association has also been observed in a study investigating the effects of prenatal stress associated with the Chernobyl disaster on adolescent cortisol levels. At the age of 14, cortisol levels were significantly higher after prenatal exposure to maternal stress from the second trimester onwards, compared with reference groups of non-exposed adolescents (Huizink et al, 2008). Inconsistency in the data may be related to differences in stress measures, given that diverging offspring cortisol findings were associated with parental stress measures at similar gestation stages. The Van den Bergh, O'Donnell, and O'Connor studies used a variety of continuous measures (State Trait Anxiety Inventory, Crown-Crisp experiential index), whereas the Huizink study compared exposed $v s$ non-exposed offspring without accounting for subjective measures of stress.

Separately, maternal prenatal cortisol has also been associated with offspring cortisol levels. Amniotic fluid cortisol, perhaps a more direct measure of fetal exposure to glucocorticoids compared with measures of cortisol from blood, predicted infant cortisol response to separationreunion stress, where infants exposed to higher levels of cortisol in utero showed higher pre-stress cortisol values and blunted response to stress exposure (O'Connor et al, 2013). Four-year-old children of mothers exhibiting higher concentrations of cortisol prenatally at 15-17 weeks of gestation had higher overall concentrations of cortisol (Gutteling et al, 2004). A separate study replicated this finding, observing that children whose mothers had higher levels of morning cortisol during pregnancy had higher circulating levels of cortisol on school days, after returning from an extended summer break (Gutteling et al, 2005). This study was particularly critical, as it demonstrated that offspring levels of cortisol were associated with maternal prenatal cortisol, as well as maternal prenatal stress; however, whether parental cortisol was associated with parental stress is unknown.

Looking forward, it will be important to clarify the relationship between parental stress and parental cortisol, which are assumed to correlate, and how these two factors associate with offspring outcomes. In the PTSD literature, evidence suggests that parents with PTSD, which is consistently associated with lower cortisol levels, transmit greater risk for PTSD by affecting offspring HPA-axis activity. However, regarding transmission of stress, without any associated psychopathology, it is still unknown how subjective feelings of stress, stressor exposure, and cortisol in parents interact to influence offspring and, moreover, what is the directionality of these interactions.

Furthermore, it will be interesting to determine whether subsequent offspring psychopathology is reflective of earlier observed HPA-axis dysregulation. Few studies have directly linked parent stress, offspring cortisol levels, and offspring behavior. One study reported that lower awakening cortisol and higher evening cortisol levels in adolescent female offspring, which was significantly associated with maternal anxiety at 12-22-week gestation, was also associated with depressive symptoms in the same offspring (Van den Bergh et al, 2008). A separate study reported that a larger cortisol response and a slower rate of behavioral recovery in neonates from a blood draw $24 \mathrm{~h}$ after birth was associated with elevated levels of maternal cortisol early in pregnancy, as well as prenatal maternal psychosocial stress throughout gestation (Davis et al, 2011).

Although circulating maternal cortisol during gestation is thought to shape development of the offspring (HPA) axis, it is important to note that only $10-20 \%$ of maternal cortisol passes to the fetus (Gitau et al, 1998). Placental $11 \beta$-hydroxysteroid dehydrogenase type $2(11 \beta$-HSD2) converts cortisol/corticosterone to inactive metabolites, regulating levels of fetal exposure to maternal cortisol levels (Wadhwa et al, 2001; Seckl, 2008). Interestingly, 11 $\beta$-HSD2 itself seems to be regulated by environmental factors, including stress. Prenatal anxiety was found to be negatively correlated with placental HSD11B2 mRNA expression (O'Donnell et al, 2012). Similarly, Holocaust survivors exhibited lower $11 \beta$-HSD2 activity compared with controls, suggesting that parental trauma could expose offspring to greater levels of cortisol exposure in utero (Yehuda et al, 2009). Altogether, changes in circulating maternal prenatal cortisol in response to stress could directly have an impact on the developing fetus, but could also alter the activity or expression of placental enzymes such as $11 \beta$-HSD2, which may then in turn regulate fetal access to parental cortisol.

\section{Epigenetics}

Although the study of offspring epigenetics is limited in humans, and largely focus on NR3C1 promoter methylation, these data are particularly exciting as the epigenome is a likely intermediary between stress-responsive biological changes in parents and biological changes, eg, HPA axis and neuroanatomical changes, in offspring. Epigenetic signatures are responsive to environmental factors, including parental stress, and are stable over long periods of time and, data now suggest, across generations to persistently modify 
TABLE 2 Epigenetic Correlates of Parental Stress in Offspring

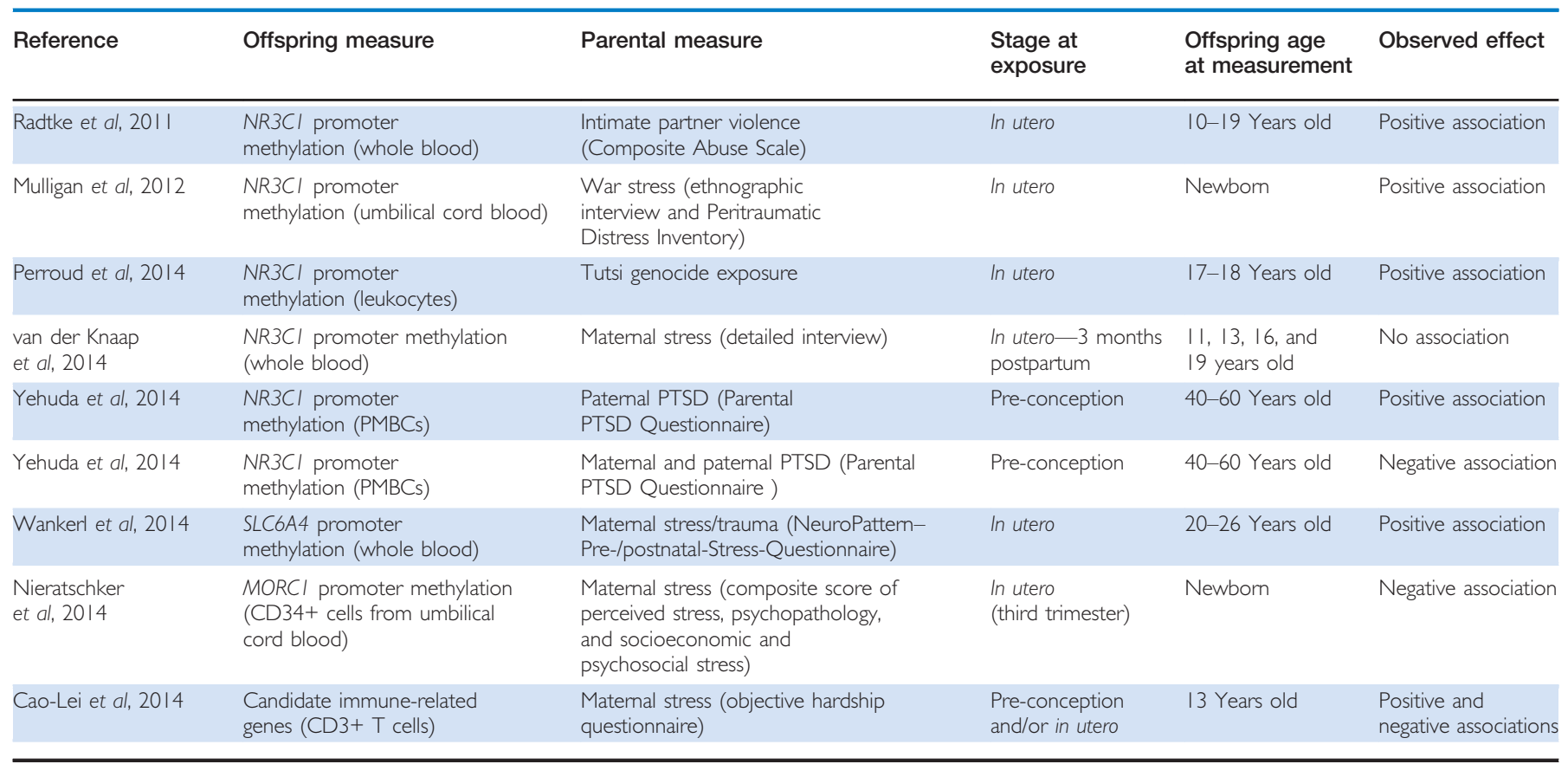

gene transcription (Weaver et al, 2004; Zhang and Meaney, 2010; Feil and Fraga, 2011). We summarize the findings of the reviewed epigenetic studies in Table 2.

Regarding the Holocaust offspring studies, the initial focus was on parental exposure and did not differentiate by parental gender. Studies demonstrating a particularly potent effect of maternal PTSD on offspring PTSD prompted a reanalysis of data to determine gender effects. Studies examining an effect of parent gender observed differential effects of maternal and paternal PTSD on both GR sensitivity and vulnerability to psychiatric disorders (Yehuda et al, 2008; Lehrner et al, 2014). Observed changes in offspring GR sensitivity may stem from parent gender-specific methylation changes to NR3C1, the gene that encodes GR. Methylation of gene promoter regions typically inhibits gene transcription (Klose and Bird, 2006). Indeed, Holocaust survivor offspring with paternal PTSD exhibited higher NR3C1 promoter methylation, whereas offspring with both maternal and paternal PTSD exhibited lower NR3C1 methylation (Yehuda et al, 2014).

Similar methylation changes associated with NR3C1 have been reported in offspring of mothers stressed during pregnancy. A positive association was observed between methylation status of the NR3C1 promoter in offspring at 10-19 years of age and maternal exposure to intimate partner violence (IPV) during pregnancy (Radtke et al, 2011). A positive correlation was also found between maternal prenatal stress associated with living in the Democratic Republic of Congo, a region plagued by ongoing war and violence, and newborn methylation in the promoter of NR3C1 in umbilical cord blood (Mulligan et al, 2012).
Perroud et al similarly found hypermethylation of the NR3C1 promoter in offspring born to Tutsi widows pregnant during the genocide of ethnic Tutsis in Rwanda (Perroud et al, 2014). However, another study has reported that there is a nonsignificant effect of perinatal stress, defined as the sum of maternal psychological problems during pregnancy or the 3 months after delivery, on NR3C1 methylation in adolescent offspring (van der Knaap et al, 2014). Incongruent findings on the methylation status of NR3C1 may be reflective of differences in methodologies or individual differences in the potential subsequent development of psychopathology in offspring. Longitudinal, comparative studies are needed to address differences in the NR3C1 methylation status, averaged and at specific $\mathrm{CpG}$ sites along $N R 3 C 1$ and NR3C1 regulatory elements, and how these differences in methylation associate with offspring outcomes.

In addition, studies have begun to investigate the offspring methylation status and gene variants of SLC6A4, the serotonin transporter gene, and an association with intergenerational stress, as SLC6A4 has been shown to moderate risk for psychopathology in the face of environmental stressors (Taylor et al, 2006; Kilpatrick et al, 2007; Grabe et al, 2009; Xie et al, 2009). Offspring born to mothers with at least one major stressful/traumatic life event during pregnancy have lower serotonin transporter mRNA levels compared with those without maternal prenatal stress. Interestingly, the authors found a negative association between the number of prenatal maternal life stressors/ trauma and SLC6A4 mRNA. Furthermore, SLC6A4 gene variants and prenatal/early adversity associated with decreased SLC6A4 expression in an additive manner, where the 
TABLE 3 Neuroanatomical Correlates of Parental Stress in Offspring

\begin{tabular}{|c|c|c|c|c|c|}
\hline Reference & Offspring measure & Parental measure & Stage at exposure & $\begin{array}{l}\text { Offspring age } \\
\text { at measurement }\end{array}$ & Observed effect \\
\hline Buss et al, 2010 & Prefrontal cortex gray matter & Maternal anxiety ( 10 -item pregnancy anxiety scale) & 19 Weeks in utero & 6-9 Years old & Negative association \\
\hline Qiu et $a l, 2013$ & Right hippocampal growth & Maternal anxiety (State Trait Anxiety Inventory) & 3 Months postnatal & 0-6 Months old & Positive association \\
\hline Qiu et $a l, 2013$ & Left hippocampal growth & Maternal anxiety (State Trait Anxiety Inventory) & 3 Months postnatal & 6 Months old & Negative association \\
\hline Qiu et $a l, 2014$ & $\begin{array}{l}\text { Dorsolateral prefrontal } \\
\text { cortex and precentral gryus }\end{array}$ & Maternal anxiety (State Trait Anxiety Inventory & 26 Weeks in utero & Newborn & $\begin{array}{l}\text { COMT SNP-moderated } \\
\text { negative association }\end{array}$ \\
\hline
\end{tabular}

lowest levels of SLC6A4 mRNA were observed in offspring exposed to greater prenatal stress carrying the short SLC6A4 allele. This decrease in SLC6A4 expression may be due to SLC6A4 methylation changes, as individuals exposed to maternal prenatal stress were found to have higher methylation levels at several SLC6A4 promoter CpG sites (Wankerl et al, 2014).

Several additional studies have observed changes in offspring gene methylation; however, an interpretation of the data from these studies is less clear. MORC1 (MORC family CW-type zinc-finger 1) promoter hypomethylation was observed in human, monkey, and rat offspring exposed to early-life stress. Although a gene-based case-control analysis demonstrated an association between MORC1 and depression, the specific role of $M O R C 1$ is largely unknown (Nieratschker et al, 2014). Furthermore, prenatal maternal objective hardship in response to the 1998 Quebec ice storm was associated with a DNA methylation changes specific to genes related to immune function in $\mathrm{CD} 3+\mathrm{T}$ cells of offspring at $\sim 13$ years of age (Cao-Lei et al, 2014).

\section{Neuroanatomical}

Over the past few years, more research has been devoted to understand the effect of parental stress on offspring neuroanatomical development. Findings related to differential offspring neuroanatomical development associated with parental stress are summarized in Table 3. Neuroimaging studies have primarily focused on changes in limbic structures responsive to stress and critical for emotion, including the prefrontal cortex and hippocampus. For instance, prenatal anxiety was reported to associate with offsprings' gray matter volume reductions in the prefrontal cortex at 6-9 years of age (Buss et al, 2010). A separate study similarly observed an effect of prenatal maternal anxiety on neonatal frontal cortical thickness that was moderated by functional variants of the catechol-O-methyltransferase $(C O M T)$ gene, which regulates catecholamine signaling and is implicated in anxiety, pain, and stress responsivity (Qiu et al, 2014). Furthermore, a strong positive association was observed between postnatal maternal anxiety and offspring right hippocampal growth and a strong negative association between postnatal maternal anxiety and offspring left hippocampal volume at 6 months of age (Pruessner et al, 2008; Qiu et al, 2013). Notably, the frontal cortex and hippocampus have been implicated in fear and stress reactivity, trait anxiety, and psychopathology, including PTSD (Rusch et al, 2001; Shin et al, 2006; Pruessner et al, 2008). In addition, frontal cortex-hippocampus signaling is thought to underlie contextual-processing, which may be disrupted in individuals with PTSD (Maren et al, 2013). These studies suggest that maternal prenatal stress could establish early structural changes in these regions that precede later clinical problems in offspring.

Interestingly, studies have also found an association between fetal synthetic glucocorticoid exposure (synthetic glucocorticoids are administered to mothers at risk for preterm delivery to promote fetal lung maturation and prevent respiratory distress syndrome) and changes in neuroanatomical development, suggesting that offspring neuroanatomical effects of prenatal anxiety could be mediated by glucocorticoid programming. Specifically, children aged 6-10 years old with synthetic fetal glucocorticoid exposure were found to have a thinner cortex primarily in the rostral anterior cingulate cortex (rACC). Separately, studies have reported that the thinner left rACC cortex was associated with offspring affective problems (Davis et al, 2013) and that prenatal synthetic glucocorticoid treatment was associated with offspring general psychiatric disturbance (Khalife et al, 2013). These data support the hypothesis that aberrant glucocorticoid signaling in the fetal environment, potentially associated with prenatal stress in mothers, alters neuroanatomical development, contributing to behavioral problems in offspring.

\section{CONCLUSIONS AND FUTURE DIRECTIONS}

At present, intergenerational transmission of stress is best studied using correlational analysis of prenatal maternal exposure to stress, subsequent maternal behavioral responses, associated changes in the gestational uterine environment, and offspring outcomes, both biological and 
psychological. Studies of newborns may shore up the best evidence for intergenerational transmission of stress, as the effects of parent behavior on offspring at this stage are presumed to be minimal. Although in utero effects have been extensively studied, the gestational environment is not the sole mechanism by which stress is transmitted, as demonstrated by the animal literature (Caldji et al, 1998; Rodgers et al, 2013; Dias and Ressler, 2014; Gapp et al, 2014). Stress could be transmitted via gametes (sperm and oocyte) and/or the early postnatal environment, aside from the gestational uterine environment. Further study investigating each of these modes of transmission, as well as how effects may be differentially transmitted by mothers and fathers, will clarify observed effects and biological correlates in offspring.

In this vein, adoptive $v s$ biological parent studies may better differentiate the relative contribution of parental stress profiles pre- and post-birth in longitudinal studies of offspring outcomes. Although this area of study is limited, one report has measured the effect of birth parent prenatal and adoptive parent postnatal depressive symptoms on offspring internalizing problems and cortisol activity (Laurent et al, 2013). Gestational surrogate studies may also clarify the role of specific variables and their contribution to transmission of stress across generations, particularly as these studies would eliminate the confound of genetics. These studies could replicate established protocols, monitoring gestational surrogate stress during pregnancy and, subsequently, parent and offspring biological measures, as well as a host of behavioral measures, longitudinally.

A considerable amount of research, undoubtedly, will continue to investigate the mechanism(s) underlying intergenerational transmission of stress. Given the significant amount of evidence that has accumulated supporting the hypothesis of parent to offspring transmission of stress, however, the field is poised to expand into multiple, additional avenues of research beyond mechanistic investigations. For instance, as more studies address the etiology and biological correlates of intergenerational stress in second-generation offspring, it will be necessary to determine how persistent these effects are and under what conditions these effects persist/decay. At least one study suggests that stress is transmitted to a third generation. Adolescent offspring of two Holocaust survivor offspring parents exhibited higher levels of ambivalent attachment style and demonstrated poorer judgment compared with peers with one Holocaust survivor offspring parent (Scharf, 2007), although a separate study found no evidence for tertiary traumatization in third-generation Holocaust survivors (Sagi-Schwartz et al, 2008). As some data from the human literature suggest that the effects of stress persist beyond the second generation, it will be critical to determine whether the same biological mechanisms that are associated with stress transmission to the second generation are also associated with transfer from the second to the third generation. Investigation of the human sperm epigenome may be the most tractable way to accomplish this goal, which has been conducted in several rodent studies (Franklin et al,
2010; Rodgers et al, 2013; Dias and Ressler, 2014; Gapp et al, 2014).

Although some rodent studies have investigated paternal sources of inherited stress, most human studies have focused on the impact of maternal stress on offspring. Some studies have suggested that maternal stress, compared with paternal stress, may be a particularly potent mediator of adverse effects in offspring (Yehuda et al, 2008). Perhaps the greater relative influence of maternal stress is because of a greater influence of the uterine environment, or perhaps this effect is confounded by increased maternal involvement in parenting. Alternatively, 'parent of origin' effects may indicate the action of imprinted genes. Genomic imprinting involves parent of origin-specific allele expression regulated by methylation. This hypothesis is consistent with some evidence that genomic imprinting moderates behavioral and cognitive functioning. A number of paternally and maternally imprinted gene loci on chromosome 15 have been implicated in several neurological and psychiatric disorders, including autism, epilepsy, schizophrenia, Angleman Syndrome, and Prader-Willi Syndrome. The occurrence or severity of these disorders depends on inheritance and imprinting status of a risk allele (Isles and Wilkinson, 2000; Reik and Walter, 2001). Regardless of whether imprinting contributes to greater influence of maternal stress on offspring, future studies should more thoroughly address the role of fathers in the transmission of stress to offspring. An obvious target is the germline of stressed men.

In addition, it will be critical to determine whether there are factors that mitigate or block intergenerational transmission of stress and, further, to identify these mitigating factors. Studies suggest that healthy relationships are protective against generational perpetuation of stress. Supportive and trusting relationships with intimate partners, high levels of maternal warmth toward children, and low levels of IPV break the cycle of abuse, where children of women who were maltreated during childhood were themselves protected from maltreatment (Jaffee et al, 2013). A recent meta-analysis indicates that safe, stable, nurturing relationships buffer intergenerational continuity of child maltreatment (Schofield et al, 2013). However, it is not clear whether these mitigating factors 'erase' or otherwise prevent the biological signatures in offspring of parent stress, eg, changes to HPA axis activity. At least one study has suggested that offspring with parental psychopathology concurrently exposed to high levels of a mitigating factor exhibited increased vagal withdrawal, assessed by respiratory sinus arrhythmia changes in response to a stressor, compared with offspring with parental psychopathology exposed to low levels of a mitigating factor (Sharp et al, 2012). Frequency of infant stroking, assessed via maternal self-report at 5 and 9 weeks after birth, was also found to modify associations between prenatal maternal depression and infant physiology and emotional reactivity, where increased maternal depression was associated with decreased vagal withdrawal and increased negative emotionality in 
infants, only in the presence of low maternal stroking, not high maternal stroking (Sharp et al, 2012).

Finally, it will be interesting to determine whether intergenerational transmission is a simple transfer of the negative consequences from stress or a way to enhance adaptive capabilities in offspring. For instance, offspring of mothers who were malnourished early in pregnancy have higher obesity rates (Roseboom et al, 2006). Perhaps, increased rates of obesity in offspring reflects changes in offspring feeding behavior and/or metabolism, which would be advantageous during famine, however, are maladaptive in contexts where food is plentiful. This debate reflects similar hypotheses regarding PTSD, where PTSD is thought to manifest from a deficit in the ability to shift behavior in response to changing contexts. The idea that intergenerational stress transmission is adaptive for offspring might explain findings where parents and offspring had opposite biological correlates of parental stress. For instance, Holocaust survivors exhibited inverse $11 \beta$-HSD2 activity and FKBP5 methylation compared with their offspring (Yehuda et al, 2009; Bierer et al, 2014; Yehuda et al, 2015). If these original effects in parents reflect exposure to extreme stress, perhaps opposite marks or activity in offspring are intended to promote resilience in a similar context.

Altogether, far less controversy regarding intergenerational transmission of stress exists today, as transmission has been documented across species, cultures, trauma types, and for a variety of psychiatric disorders. Conflict, rather, stems from the etiology of this transmission. Although investigations into the intergenerational transmission of stress in humans will continue to be challenged by constraints related to the study of human subjects, the interpretation of these studies will be boosted by biological manipulations in animal studies, the use of conservative statistical tests, large cohorts of human subjects, and the use of identified gold standard techniques. While we propose a few directed lines of inquiry, including the investigation of the role of fathers, imprinting, mitigating factors, and the perpetuation of stress across multiple generations, the field is ripe for addressing a multitude of hypotheses.

\section{FUNDING AND DISCLOSURE}

The authors declare no conflict of interest.

\section{ACKNOWLEDGMENTS}

We thank Dr Linda Bierer for help in reviewing the manuscript and Max Ehrlich for contributing to the design of Figure 1.

\section{REFERENCES}

Bandura A. Social learning theory. Prentice Hall: Englewood Cliffs, N.J., 1977. Barker DJ (1990). The fetal and infant origins of adult disease. Br Med J 301: 1111. Barker DJ (1998). In utero programming of chronic disease. Clin Sci (Lond) 95: $115-128$.
Berkowitz GS, Wolff MS, Janevic TM, Holzman IR, Yehuda R, Landrigan PJ (2003). The World Trade Center disaster and intrauterine growth restriction. JAMA 290: 595-596.

Bierer LM, Bader HN, Daskalakis NP, Lehrner AL, Makotkine I, Seckl JR et al (2014). Elevation of 11 beta-hydroxysteroid dehydrogenase type 2 activity in Holocaust survivor offspring: evidence for an intergenerational effect of maternal trauma exposure. Psychoneuroendocrinology 48: 1-10.

Buss C, Davis EP, Hobel CJ, Sandman CA (2011). Maternal pregnancy-specific anxiety is associated with child executive function at 6-9 years age. Stress 14: 665-676.

Buss C, Davis EP, Muftuler LT, Head K, Sandman CA (2010). High pregnancy anxiety during mid-gestation is associated with decreased gray matter density in 6-9-year-old children. Psychoneuroendocrinology 35: 141-153.

Caldji C, Tannenbaum B, Sharma S, Francis D, Plotsky PM, Meaney MJ (1998). Maternal care during infancy regulates the development of neural systems mediating the expression of fearfulness in the rat. Proc Natl Acad Sci USA 95 5335-5340.

Cao-Lei L, Massart R, Suderman MJ, Machnes Z, Elgbeili G, Laplante DP et al (2014). DNA methylation signatures triggered by prenatal maternal stress exposure to a natural disaster: Project Ice Storm. PLoS One 9: e107653.

Cederbaum JA, Putnam-Hornstein E, King B, Gilbert K, Needell B (2013). Infant birth weight and maltreatment of adolescent mothers. Am J Prev Med 45: 197-201.

Christiaens I, Hegadoren K, Olson DM (2015). Adverse childhood experiences are associated with spontaneous preterm birth: a case-control study. BMC Med 13: 124.

Cortez MA, Bueso-Ramos C, Ferdin J, Lopez-Berestein G, Sood AK, Calin GA (2011). MicroRNAs in body fluids-the mix of hormones and biomarkers. Nat Rev Clin Oncol 8: 467-477.

Coussons-Read, Lobel M, Carey JC, Kreither MO, D'Anna K, Argys L et al (2012). The occurrence of preterm delivery is linked to pregnancy-specific distress and elevated inflammatory markers across gestation. Brain Behav Immun 26: 650-659

Davis EP, Glynn LM, Waffarn F, Sandman CA (2011). Prenatal maternal stress programs infant stress regulation. J Child Psychol Psychiatry 52: 119-129.

Davis EP, Sandman CA, Buss C, Wing DA, Head K (2013). Fetal glucocorticoid exposure is associated with preadolescent brain development. Biol Psychiatry 74: 647-655.

Daxinger L, Whitelaw E (2012). Understanding transgenerational epigenetic inheritance via the gametes in mammals. Nat Rev Genet 13: 153-162.

Delahanty DL, Raimonde AJ, Spoonster E (2000). Initial posttraumatic urinary cortisol levels predict subsequent PTSD symptoms in motor vehicle accident victims. Biol Psychiatry 48: 940-947.

Dias BG, Ressler KJ (2014). Parental olfactory experience influences behavior and neural structure in subsequent generations. Nat Neurosci 17: 89-96.

Feil R, Fraga MF (2011). Epigenetics and the environment: emerging patterns and implications. Nat Rev Genet 13: 97-109.

Flory JD, Bierer LM, Yehuda R (2011). Maternal exposure to the holocaust and health complaints in offspring. Dis Markers 30: 133-139.

Franklin TB, Russig H, Weiss IC, Graff J, Linder N, Michalon A et al (2010). Epigenetic transmission of the impact of early stress across generations. Biol Psychiatry 68: 408-415.

Gangi S, Talamo A, Ferracuti S (2009). The long-term effects of extreme war-related trauma on the second generation of Holocaust survivors. Violence Vict 24: $687-700$

Gapp K, Jawaid A, Sarkies P, Bohacek J, Pelczar P, Prados J et al (2014). Implication of sperm RNAs in transgenerational inheritance of the effects of early trauma in mice. Nat Neurosci 17: 667-669.

Gitau R, Cameron A, Fisk NM, Glover V (1998). Fetal exposure to maternal cortisol. Lancet 352: 707-708.

Glover V (1997). Maternal stress or anxiety in pregnancy and emotional development of the child. Br J Psychiatry 171: 105-106.

Grabe HJ, Spitzer C, Schwahn C, Marcinek A, Frahnow A, Barnow S et al (2009). Serotonin transporter gene (SLC6A4) promoter polymorphisms and the susceptibility to posttraumatic stress disorder in the general population. Am J Psychiatry 166: 926-933.

Graves CN, Eiler H (1979). Cortisol content of semen and the effect of exogenous cortisol on the concentration of cortisol and minerals ( $\mathrm{Ca}, \mathrm{Mg}, \mathrm{K}$ and $\mathrm{Na}$ ) in semen and blood plasma of bulls. Biol Reprod 21: 1225-1229.

Gutteling BM, de Weerth C, Buitelaar JK (2004). Maternal prenatal stress and 4-6 year old children's salivary cortisol concentrations pre- and post-vaccination. Stress 7: 257-260.

Gutteling BM, de Weerth C, Buitelaar JK (2005). Prenatal stress and children's cortisol reaction to the first day of school. Psychoneuroendocrinology 30: 541-549. 
Hammen C (1991). Generation of stress in the course of unipolar depression. J Abnorm Psychol 100: 555-561.

Honda M, Kuwano Y, Katsuura-Kamano S, Kamezaki Y, Fujita K, Akaike Y et al (2013). Chronic academic stress increases a group of microRNAs in peripheral blood. PLoS One 8: e75960.

Huizink AC, Bartels M, Rose RJ, Pulkkinen L, Eriksson CJ, Kaprio J (2008). Chernobyl exposure as stressor during pregnancy and hormone levels in adolescent offspring. J Epidemiol Community Health 62: e5.

Huizink AC, Dick DM, Sihvola E, Pulkkinen L, Rose RJ, Kaprio J (2007). Chernobyl exposure as stressor during pregnancy and behaviour in adolescent offspring. Acta Psychiatr Scand 116: 438-446.

Isles AR, Wilkinson LS (2000). Imprinted genes, cognition and behaviour. Trends Cogn Sci 4: 309-318.

Issler O, Chen A (2015). Determining the role of microRNAs in psychiatric disorders. Nat Rev Neurosci 16: 201-212.

Jaffee SR, Bowes L, Ouellet-Morin I, Fisher HL, Moffitt TE, Merrick MT et al (2013). Safe, stable, nurturing relationships break the intergenerational cycle of abuse: a prospective nationally representative cohort of children in the United Kingdom. $\checkmark$ Adolesc Health 53: S4-S10.

Jerskey BA, Panizzon MS, Jacobson KC, Neale MC, Grant MD, Schultz M et al (2010). Marriage and divorce: a genetic perspective. Pers Individ Dif 49: 473-478.

Khalife N, Glover V, Taanila A, Ebeling H, Jarvelin MR, Rodriguez A (2013). Prenatal glucocorticoid treatment and later mental health in children and adolescents. PLoS One 8: e81394.

Kilpatrick DG, Koenen KC, Ruggiero KJ, Acierno R, Galea S, Resnick HS et al (2007). The serotonin transporter genotype and social support and moderation of posttraumatic stress disorder and depression in hurricane-exposed adults. Am J Psychiatry 164: 1693-1699.

Klose RJ, Bird AP (2006). Genomic DNA methylation: the mark and its mediators. Trends Biochem Sci 31: 89-97.

Laplante DP, Barr RG, Brunet A, Galbaud du Fort G, Meaney ML, Saucier JF et al (2004). Stress during pregnancy affects general intellectual and language functioning in human toddlers. Pediatr Res 56: 400-410.

Laurent HK, Leve LD, Neiderhiser JM, Natsuaki MN, Shaw DS, Harold GT et al (2013). Effects of prenatal and postnatal parent depressive symptoms on adopted child HPA regulation: independent and moderated influences. Dev Psychol 49: 876-886.

Lederman SA, Rauh V, Weiss L, Stein JL, Hoepner LA, Becker M et al (2004). The effects of the World Trade Center event on birth outcomes among term deliveries at three lower Manhattan hospitals. Environ Health Perspect 112: 1772-1778.

Lehrner A, Bierer LM, Passarelli V, Pratchett LC, Flory JD, Bader HN et al (2014). Maternal PTSD associates with greater glucocorticoid sensitivity in offspring of Holocaust survivors. Psychoneuroendocrinology 40: 213-220.

Liu D, Diorio J, Tannenbaum B, Caldji C, Francis D, Freedman A et al (1997). Maternal care, hippocampal glucocorticoid receptors, and hypothalamic-pituitaryadrenal responses to stress. Science 277: 1659-1662.

Maren S, Phan KL, Liberzon I (2013). The contextual brain: implications for fear conditioning, extinction and psychopathology. Nat Rev Neurosci 14: 417-428.

McEwen BS (2006). Protective and damaging effects of stress mediators: central role of the brain. Dialogues Clin Neurosci 8: 367-381.

Mennes M, Stiers P, Lagae L, Van den Bergh B (2006). Long-term cognitive sequelae of antenatal maternal anxiety: involvement of the orbitofrontal cortex. Neurosci Biobehav Rev 30: 1078-1086.

Mulligan CJ, D'Errico NC, Stees J, Hughes DA (2012). Methylation changes at NR3C1 in newborns associate with maternal prenatal stress exposure and newborn birth weight. Epigenetics 7: 853-857.

Nieratschker V, Massart R, Gilles M, Luoni A, Suderman MJ, Krumm B et al (2014). MORC1 exhibits cross-species differential methylation in association with early life stress as well as genome-wide association with MDD. Transl Psychiatry 4: e429.

O'Connor TG, Ben-Shlomo Y, Heron J, Golding J, Adams D, Glover V (2005). Prenatal anxiety predicts individual differences in cortisol in pre-adolescent children. Biol Psychiatry 58: 211-217.

O'Connor TG, Bergman K, Sarkar P, Glover V (2013). Prenatal cortisol exposure predicts infant cortisol response to acute stress. Dev Psychobiol 55: 145-155.

O'Connor TG, Hetherington EM, Reiss D, Plomin R (1995). A twin-sibling study of observed parent-adolescent interactions. Child Dev 66: 812-829.

O'Connor TG, Heron J, Golding J, Beveridge M, Glover V (2002). Maternal antenatal anxiety and children's behavioural/emotional problems at 4 years. Report from the Avon Longitudinal Study of Parents and Children. Br J Psychiatry 180: 502-508.

O'Donnell KJ, Bugge Jensen A, Freeman L, Khalife N, O'Connor TG, Glover V (2012). Maternal prenatal anxiety and downregulation of placental 11beta-HSD2. Psychoneuroendocrinology 37: 818-826.

O'Donnell KJ, Glover V, Jenkins J, Browne D, Ben-Shlomo Y, Golding J et al (2013). Prenatal maternal mood is associated with altered diurnal cortisol in adolescence. Psychoneuroendocrinology 38: 1630-1638.
Perroud N, Rutembesa E, Paoloni-Giacobino A, Mutabaruka J, Mutesa L, Stenz L et al (2014). The Tutsi genocide and transgenerational transmission of maternal stress: epigenetics and biology of the HPA axis. World J Biol Psychiatry 15: 334-345

Pineles SL, Rasmusson AM, Yehuda R, Lasko NB, Macklin ML, Pitman RK et al (2013). Predicting emotional responses to potentially traumatic events from preexposure waking cortisol levels: a longitudinal study of police and firefighters. Anxiety Stress Coping 26: 241-253.

Pruessner JC, Dedovic K, Khalili-Mahani N, Engert V, Pruessner M, Buss C et al (2008). Deactivation of the limbic system during acute psychosocial stress: evidence from positron emission tomography and functional magnetic resonance imaging studies. Biol Psychiatry 63: 234-240.

Qiu A, Rifkin-Graboi A, Chen H, Chong YS, Kwek K, Gluckman PD et al (2013). Maternal anxiety and infants' hippocampal development: timing matters. Trans/ Psychiatry 3: e306.

Qiu A, Tuan TA, Ong ML, Li Y, Chen H, Rifkin-Graboi A et al (2014). COMT haplotypes modulate associations of antenatal maternal anxiety and neonatal cortical morphology. Am J Psychiatry 172: 163-172.

Radtke KM, Ruf M, Gunter HM, Dohrmann K, Schauer M, Meyer A et al (2011). Transgenerational impact of intimate partner violence on methylation in the promoter of the glucocorticoid receptor. Transl Psychiatry 1: e21.

Reik W, Walter J (2001). Genomic imprinting: parental influence on the genome. Nat Rev Genet 2: 21-32.

Roberts AL, Galea S, Austin SB, Cerda M, Wright RJ, Rich-Edwards JW et al (2012). Posttraumatic stress disorder across two generations: concordance and mechanisms in a population-based sample. Biol Psychiatry 72: 505-511.

Rodgers AB, Morgan CP, Bronson SL, Revello S, Bale TL (2013). Paternal stress exposure alters sperm microRNA content and reprograms offspring HPA stress axis regulation. J Neurosci 33: 9003-9012.

Roseboom T, de Rooij S, Painter R (2006). The Dutch famine and its long-term consequences for adult health. Early Hum Dev 82: 485-491.

Rusch BD, Abercrombie HC, Oakes TR, Schaefer SM, Davidson RJ (2001). Hippocampal morphometry in depressed patients and control subjects: relations to anxiety symptoms. Biol Psychiatry 50: 960-964.

Sagi-Schwartz A, van IMH, Bakermans-Kranenburg MJ (2008). Does intergenerational transmission of trauma skip a generation? No meta-analytic evidence for tertiary traumatization with third generation of Holocaust survivors. Attach Hum Dev 10: 105-121.

Scharf M (2007). Long-term effects of trauma: psychosocial functioning of the second and third generation of Holocaust survivors. Dev Psychopathol 19: 603-622.

Schofield TJ, Lee RD, Merrick MT (2013). Safe, stable, nurturing relationships as a moderator of intergenerational continuity of child maltreatment: a meta-analysis. $J$ Adolesc Health 53: S32-S38.

Seckl JR (2008). Glucocorticoids, developmental 'programming' and the risk of affective dysfunction. Prog Brain Res 167: 17-34.

Selye H. The stress of life. McGraw-Hill: New York, 1956.

Sharp H, Pickles A, Meaney M, Marshall K, Tibu F, Hill J (2012). Frequency of infant stroking reported by mothers moderates the effect of prenatal depression on infant behavioural and physiological outcomes. PLoS One 7: e45446.

Shin LM, Rauch SL, Pitman RK (2006). Amygdala, medial prefrontal cortex, and hippocampal function in PTSD. Ann N Y Acad Sci 1071: 67-79.

Solomon Z, Kotler M, Mikulincer M (1988). Combat-related posttraumatic stress disorder among second-generation Holocaust survivors: preliminary findings. Am J Psychiatry 145: 865-868.

Spiegelman A. Maus II : a survivor's tale : and here my troubles began. 1st edn. Pantheon Books: New York, 1991.

Stahn C, Lowenberg M, Hommes DW, Buttgereit F (2007). Molecular mechanisms of glucocorticoid action and selective glucocorticoid receptor agonists. Mol Cell Endocrinol 275: 71-78.

Stratholt ML, Donaldson EM, Liley NR (1997). Stress induced elevation of plasma cortisol in adult female coho salmon (Oncorhynchus kisutch), is reflected in egg cortisol content, but does not appear to affect early development. Aquaculture 158: $141-153$

Taylor SE, Way BM, Welch WT, Hilmert CJ, Lehman BJ, Eisenberger NI (2006). Early family environment, current adversity, the serotonin transporter promoter polymorphism, and depressive symptomatology. Biol Psychiatry 60: 671-676.

Van den Bergh BR, Mennes M, Oosterlaan J, Stevens V, Stiers P, Marcoen A et al (2005). High antenatal maternal anxiety is related to impulsivity during performance on cognitive tasks in 14- and 15-year-olds. Neurosci Biobehav Rev 29: 259-269.

Van den Bergh BR, Van Calster B, Smits T, Van Huffel S, Lagae L (2008). Antenatal maternal anxiety is related to HPA-axis dysregulation and self-reported depressive symptoms in adolescence: a prospective study on the fetal origins of depressed mood. Neuropsychopharmacology 33: 536-545. 
van der Knaap LJ, Riese H, Hudziak JJ, Verbiest MM, Verhulst FC, Oldehinkel AJ et al (2014). Glucocorticoid receptor gene (NR3C1) methylation following stressful events between birth and adolescence. The TRAILS study. Trans/ Psychiatry 4: e381.

Vostanis P, Graves A, Meltzer H, Goodman R, Jenkins R, Brugha T (2006). Relationship between parental psychopathology, parenting strategies and child mental health-findings from the GB national study. Soc Psychiatry Psychiatr Epidemio/ 41: 509-514.

Wadhwa PD, Culhane JF, Rauh V, Barve SS (2001). Stress and preterm birth: neuroendocrine, immune/inflammatory, and vascular mechanisms. Matern Child Health J 5: 119-125.

Wadhwa PD, Sandman CA, Porto M, Dunkel-Schetter C, Garite TJ (1993). The association between prenatal stress and infant birth weight and gestational age at birth: a prospective investigation. Am J Obstet Gynecol 169: 858-865.

Wankerl M, Miller R, Kirschbaum C, Hennig J, Stalder T, Alexander N (2014). Effects of genetic and early environmental risk factors for depression on serotonin transporter expression and methylation profiles. Trans/ Psychiatry 4: e402.

Watson JB, Mednick SA, Huttunen M, Wang X (1999). Prenatal teratogens and the development of adult mental illness. Dev Psychopathol 11: 457-466.

Weaver IC, Cervoni N, Champagne FA, D'Alessio AC, Sharma S, Seckl JR et al (2004). Epigenetic programming by maternal behavior. Nat Neurosci 7: 847-854.

Xie P, Kranzler HR, Poling J, Stein MB, Anton RF, Brady K et al (2009). Interactive effect of stressful life events and the serotonin transporter 5-HTTLPR genotype on posttraumatic stress disorder diagnosis in 2 independent populations. Arch Gen Psychiatry 66: 1201-1209.

Yehuda R (2002). Post-traumatic stress disorder. N Engl J Med 346: 108-114.

Yehuda R, Bell A, Bierer LM, Schmeidler J (2008). Maternal, not paternal, PTSD is related to increased risk for PTSD in offspring of Holocaust survivors. J Psychiatr Res 42: 1104-1111.

Yehuda R, Bierer LM (2009). The relevance of epigenetics to PTSD: implications for the DSM-V. J Trauma Stress 22: 427-434.

Yehuda R, Bierer LM, Andrew R, Schmeidler J, Seckl JR (2009). Enduring effects of severe developmental adversity, including nutritional deprivation, on cortisol metabolism in aging Holocaust survivors. J Psychiatr Res 43: $877-883$
Yehuda R, Bierer LM, Schmeidler J, Aferiat DH, Breslau I, Dolan S (2000). Low cortisol and risk for PTSD in adult offspring of holocaust survivors. Am J Psychiatry 157: 1252-1259.

Yehuda R, Daskalakis NP, Bierer LM, Bader HN, Klengel T, Holsboer F et al (2015). Holocaust exposure induced intergenerational effects on FKBP5 methylation. Biol Psychiatry (e-pub ahead of print).

Yehuda R, Daskalakis NP, Lehrner A, Desarnaud F, Bader HN, Makotkine I et al (2014). Influences of maternal and paternal PTSD on epigenetic regulation of the glucocorticoid receptor gene in Holocaust survivor offspring. Am J Psychiatry 171: 872-880

Yehuda R, Engel SM, Brand SR, Seckl J, Marcus SM, Berkowitz GS (2005). Transgenerational effects of posttraumatic stress disorder in babies of mothers exposed to the World Trade Center attacks during pregnancy. J Clin Endocrinol Metab 90: 4115-4118.

Yehuda R, Halligan SL, Bierer LM (2001). Relationship of parental trauma exposure and PTSD to PTSD, depressive and anxiety disorders in offspring. J Psychiatr Res 35: $261-270$.

Yehuda R, Halligan SL, Bierer LM (2002). Cortisol levels in adult offspring of Holocaust survivors: relation to PTSD symptom severity in the parent and child. Psychoneuroendocrinology 27: 171-180.

Yehuda R, McFarlane AC (1995). Conflict between current knowledge about posttraumatic stress disorder and its original conceptual basis. Am J Psychiatry 152: 1705-1713.

Yehuda R, Schmeidler J, Wainberg M, Binder-Brynes K, Duvdevani T (1998). Vulnerability to posttraumatic stress disorder in adult offspring of Holocaust survivors. Am J Psychiatry 155: 1163-1171.

Yehuda R, Teicher MH, Seckl JR, Grossman RA, Morris A, Bierer LM (2007). Parental posttraumatic stress disorder as a vulnerability factor for low cortisol trait in offspring of holocaust survivors. Arch Gen Psychiatry 64 1040-1048.

Yonkers KA, Smith MV, Forray A, Epperson CN, Costello D, Lin H et al (2014). Pregnant women with posttraumatic stress disorder and risk of preterm birth. JAMA Psychiatry 71: 897-904.

Zhang TY, Meaney MJ (2010). Epigenetics and the environmental regulation of the genome and its function. Annu Rev Psychol 61: 439-466, C1-3. 